\title{
Exercise signalling to glucose transport in skeletal muscle
}

\author{
Erik A. Richter*, Jakob N. Nielsen, Sebastian B. Jørgensen, Christian Frøsig, Jesper B. Birk \\ and Jørgen F. P. Wojtaszewski \\ Copenhagen Muscle Research Centre, Department of Human Physiology, Institute of Exercise and Sport Sciences, \\ University of Copenhagen, Universitetsparken 13, 2100 Copenhagen $\emptyset$, Denmark
}

\begin{abstract}
Contraction-induced glucose uptake in skeletal muscle is mediated by an insulin-independent mechanism that leads to translocation of the GLUT4 glucose transporter to the muscle surface membrane from an intracellular storage site. Although the signalling events that increase glucose transport in response to muscle contraction are not fully elucidated, the aim of the present review is to briefly present the current understanding of the molecular signalling mechanisms involved. Glucose uptake may be regulated by $\mathrm{Ca}^{2+}$-sensitive contraction-related mechanisms, possibly involving $\mathrm{Ca}^{2+} /$ calmodulin-dependent protein kinase II and some isoforms of protein kinase $\mathrm{C}$. In addition, glucose transport may be regulated by mechanisms that reflect the metabolic status of the muscle, probably involving the 5'AMP-activated protein kinase. Furthermore, the p38 mitogen-activated protein kinase may be involved in activating the GLUT4 translocated to the surface membrane. Nevertheless, the picture is incomplete, and fibre type differences also seem to be involved.
\end{abstract}

Contraction: AMP-activated protein kinase: $\mathrm{Ca}^{2+} /$ calmodulin-dependent protein kinase: Protein kinase C: p38 Mitogen-activated protein kinase

Glucose uptake in skeletal muscle during exercise involves acceleration of several processes compared with the resting state. These processes include increased supply of glucose obtained via increased capillary perfusion, increased membrane transport capacity obtained via translocation of GLUT4 from a designated intracellular storage compartment to the plasma membrane and transverse tubules (Ploug et al. 1998) and possibly via increased intrinsic activity of GLUT4 (for review, see Furtado et al. 2003). Finally, the metabolism of the incoming glucose has to be increased. The present review will concentrate on mechanisms involved in increasing muscle membrane glucose transport capacity during exercise or muscle contraction.

A helpful way of considering the regulation of muscle glucose transport may be in terms of two levels of regulation, as proposed by Ihlemann et al. (1999b). One level is related to the stimulation frequency of the motor nerve and thereby the average intramyocellular $\mathrm{Ca}^{2+}$ concentration. This signalling pathway may involve $\mathrm{Ca}^{2+} /$ calmodulindependent protein kinase $(\mathrm{CaMK})$ and some isoforms of protein kinase $\mathrm{C}$ (PKC; Fig. 1). The second level of control is thought to be related to the actual metabolic status of the muscle cell during contraction, as reflected by energy charge, ion balance, $\mathrm{pH}$ and substrate levels, and may implicate the 5'AMP-activated protein kinase (AMPK). Thus, glucose transport during contraction may be regulated by several different mechanisms that are probably partly redundant.

\section{Calcium-related mechanisms}

Early evidence for a stimulatory role of intracellular $\mathrm{Ca}^{2+}$ in increasing muscle glucose transport comes from studies showing that a pharmacologically-induced increase in myoplasmic $\mathrm{Ca}^{2+}$ concentration increases glucose transport in non-contraction muscle (Holloszy \& Narahara, 1967; Youn et al. 1991). Based on data obtained in electrically-stimulated frog skeletal muscle, Holloszy \& Narahara (1965) have proposed that glucose transport during contraction is a function determined only by the stimulation frequency of the muscle. In partial agreement

\footnotetext{
Abbreviations: AICAR, 5-aminoimidazole-4-carboxamide-riboside; AMPK, AMP-activated protein kinase; aPKC, atypical PKC; CaMK, Ca ${ }^{2+}$ calmodulin-dependent protein kinase; MAPK, mitogen-activated protein kinase; PKC, protein kinase C.

*Corresponding author: Dr Erik A. Richter, fax +45353216 00, email erichter@aki.ku.dk
} 


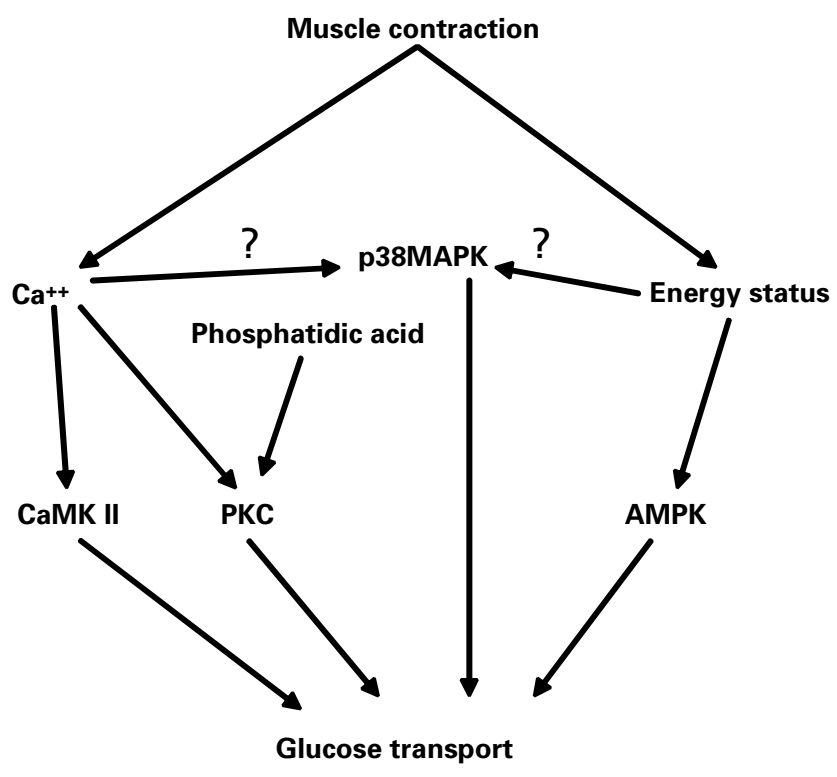

Fig. 1. Schematic presentation of hypothetical activation pathways for glucose transport in contracting skeletal muscle. The calciumactivated pathway may involve calmodulin kinase II (CaMKII), protein kinase $\mathrm{C}$ (PKC) isoforms and other still unknown signals, whereas the AMP-activated protein kinase (AMPK) is activated according to the energy status of the muscle. MAPK, mitogenactivated protein kinase.

with these studies, results in incubated rat skeletal muscle have shown that changing the stimulation frequency, while keeping the mechanical output of the muscle constant, to some extent changes glucose transport in parallel with the changes in stimulation frequency (Ihlemann et al. 2000). Nevertheless, as glucose transport and stimulation frequency only partially covary, these latter studies also suggest that it is not only the stimulation frequency (and by inference the intracellular $\mathrm{Ca}^{2+}$ concentration) that determines the glucose transport capacity of mammalian skeletal muscle.

The signals downstream from $\mathrm{Ca}^{2+}$ that are involved in contraction-induced glucose transport have not been identified with certainty, but candidates include CaMKII. An increase in intracellular $\mathrm{Ca}^{2+}$ promotes interaction of calmodulin with a diverse group of cellular proteins, including the calmodulin kinases, of which several isoforms exist (Yokokura et al. 1997). In fact, calmodulin inhibitors have previously been shown to reduce insulinstimulated glucose transport in muscle (Youn et al. 1994). Experiments with calmodulin inhibitors have revealed inhibition of glucose transport during muscle contraction as well as decreased insulin-induced muscle glucose transport (Ihlemann et al. 1999a), making it hard to draw firm conclusions about the role of calmodulin during contractions, since the effect of the inhibitors might reflect an effect on muscle glucose transport that is not specific to contraction. On the other hand, a recent report (Wright et al. 2004) suggests that the CaMKII inhibitor KN62 decreases contraction-induced glucose transport by about $50 \%$ in incubated rat epitrochlearis muscle, but there is no mention of whether the inhibitor also affects muscle force production. In addition, it has been reported recently that CaMKII autonomous activity is increased in human skeletal muscle during exercise (Rose \& Hargreaves, 2003). Thus, at present there is increasing evidence implicating calmodulin or calmodulin-dependent kinase in contractioninduced glucose transport, but more definitive evidence may be derived from experiments using molecular deletion or overexpression of the enzyme.

Other potential candidates for downstream targets of $\mathrm{Ca}^{2+}$ include PKC and, in particular, the $\mathrm{Ca}^{2+}$-sensitive conventional protein kinase PKC isoforms $\alpha, \beta$ and $\gamma$ because of the following observations. Several years ago it was shown that total PKC activity to some extent translocates from the cytosol to the particulate fraction (suggesting activation) with muscle contraction (Richter et al. 1987). Furthermore, muscle contraction also increases the concentration of diacylglycerol (Cleland et al. 1989), which activates the conventional and novel isoforms of PKC. In addition, administration of the PKC inhibitor calphostin $\mathrm{C}$ to incubated and perfused muscle has been shown to partially inhibit contraction-induced glucose transport in a fibre-type specific manner (Wojtaszewski et al. 1998; Ihlemann et al. 1999a). Since calphostin $\mathrm{C}$ is thought to block primarily diacylglycerolsensitive PKC, these findings implicate the conventional and/or novel isoforms in contraction-induced glucose transport, although a blocking effect of calphostin $\mathrm{C}$ on atypical PKC (aPKC) also cannot be ruled out (RV Farese, personal communication). Recent evidence has implicated several of the PKC isoforms (and especially the aPKC) in insulin-induced glucose transport in several cell types, including skeletal muscle (Farese, 2002). Furthermore, it has been shown recently that treadmill running in mice (Chen et al. 2002) and bicycle exercise in human subjects (Beeson et al. 2003; Nielsen et al. 2003) increases the activity of aPKC and abundance and phosphorylation of $\mathrm{PKC} \zeta$ in the membrane fraction (Perrini et al. 2004) in skeletal muscle. The mechanism for the increase in aPKC activity might be the contraction-associated increase in phosphatidic acid (Cleland et al. 1989), which is known to activate aPKC (Farese, 2002). Although such findings in vivo do not necessarily indicate that increased activity of aPKC is involved in exercise-induced glucose transport, the findings are compatible with such a role for aPKC in view of their apparent role in insulin-induced glucose transport. Based on this factor and the previously mentioned in vitro indications for PKC involvement in glucose transport, further research into the role of PKC activity in contractioninduced muscle glucose transport is warranted.

\section{Mechanisms related to the metabolic status of the muscle}

If glucose transport could only be activated by Ca-sensitive mechanisms associated with excitation-contraction coupling, the regulatory and adaptive capacity of the system would be limited. Thus, contraction-induced muscle glucose transport is probably also regulated by feedback signals related to the metabolic status of the muscle. Evidence of feedback regulation of muscle glucose 
transport in both fast- and slow-twitch muscle has been provided by Ihlemann et al. $(1999 b, 2001)$, who varied the load on the muscle while stimulating the muscle electrically at a constant frequency. Muscle glucose transport was found to vary closely with the mechanical output of the contracting muscle and thus by the metabolic stress (as evaluated by nucleotide status). Interestingly, glucose transport also correlated well with the activity of AMPK. Since AMPK is regarded as a fuel sensor activated by a decrease in creatine phosphate:creatine and ATP:AMP, 5'AMPK is a good candidate for a kinase that may regulate muscle glucose transport during cellular stress, such as intense muscle contraction (Winder \& Hardie, 1999; Fig. 1).

Another level of feedback regulation may be related to conditions in which glycogen (the major carbohydrate source) is scarce. It has been repeatedly shown, both in vivo and in vitro, that when muscle glycogen is low glucose uptake is increased during contraction (Gollnick et al. 1981; Richter \& Galbo, 1986; Hespel \& Richter, 1990; Wojtaszewski et al. 2003). The mechanism for this phenomenon is related to increased glucose transport and GLUT4 translocation when precontraction muscle glycogen is low (Derave et al. 1999) and may be coupled to AMPK, since both at rest and during exercise AMPK activity is higher in muscles with a low glycogen content than in those with a high glycogen content (Derave et al. 2000; Wojtaszewski et al. 2002, 2003).

\section{AMP-activated protein kinase}

AMPK can be activated by 5-aminoimidazole-4-carboxamide-riboside (AICAR) in resting muscle and AICAR also results in activation of muscle glucose transport independently of insulin (Merrill et al. 1997; Hayashi et al. 1998). Since AMPK is also activated in skeletal muscle during contraction or exercise in both rodent (Winder \& Hardie, 1996; Vavvas et al. 1997; Hayashi et al. 1998; Derave et al. 2000; Ihlemann et al. 2001) and human skeletal muscle (Chen et al. 2000; Wojtaszewski et al. 2000, 2003; Nielsen et al. 2002), it has been suggested that AMPK may be involved in increasing glucose transport during muscle contraction. In human subjects exercising at $70 \%$ of their $\mathrm{V}_{\mathrm{O}_{2} \max }$ and having either high or low muscle glycogen levels muscle $\alpha 2$ AMPK activity and muscle glucose uptake are higher when glycogen levels are low than when they are high (Wojtaszewski et al. 2003). Such covariation of mean values may be interpreted as supportive evidence for the notion that AMPK is involved in exercise-induced glucose uptake, but correlative evidence is not necessarily causative. Further correlative evidence between exerciseinduced glucose uptake and AMPK activity can be sought in patients with McArdle's disease (glycogen myophosphorylase deficiency). In these patients exercise-induced glucose uptake is exaggerated compared with control subjects working at the same absolute work intensity (Nielsen et al. 2002). If AMPK has a regulatory role in glucose uptake it could be hypothesized that AMPK activity would be enhanced during exercise in these patients. It has been found that glucose utilization, $\alpha 2$ MPK activity and $\beta$ acetyl-CoA carboxylase-Ser ${ }^{221}$ phosphorylation are enhanced in patients with McArdle's disease during $20 \mathrm{~min}$ of exercise when compared with the healthy control subjects exercising at the same absolute exercise load. This finding is consistent with a role for AMPK in exercise-induced glucose uptake (Nielsen et al. 2002).

There is also some evidence against a role of AMPK in contraction-induced muscle glucose transport. For instance, in slow-twitch rat muscle dissociation between AMPK activity and glucose transport during electricallyinduced contractions has been clearly shown (Fig. 2; Derave et al. 2000), suggesting that AMPK activity is not closely related to glucose transport. Furthermore, no significant correlation of the individual data points for glucose utilization and AMPK activation was found in the study of the patients with McArdle's disease (Nielsen et al. 2002) or in the experiment with high and low muscle glycogen levels (Wojtaszewski et al. 2003). Thus, mean values for exercise-induced AMPK activity and glucose utilization covary during exercise in two different human experimental models but the lack of significant correlations of individual values may indicate that there are other factors controlling exercise-induced glucose uptake.

However, these types of experiments, although physiologically important, do not provide definitive evidence of the involvement of AMPK in exercise-induced muscle glucose uptake. Using genetically-manipulated organisms or cell lines may provide more specific information; however a major question relating to these systems is the extent to which they reflect the situation in an exercising individual. Nevertheless, overexpression of a dominant negative form of AMPK (a dead kinase) in mouse muscle has been shown to abolish the AICAR- and hypoxiainduced glucose transport ( $\mathrm{Mu}$ et al. 2001). This finding indicates that hypoxia and AICAR stimulation may use the same signalling pathway through AMPK to increase

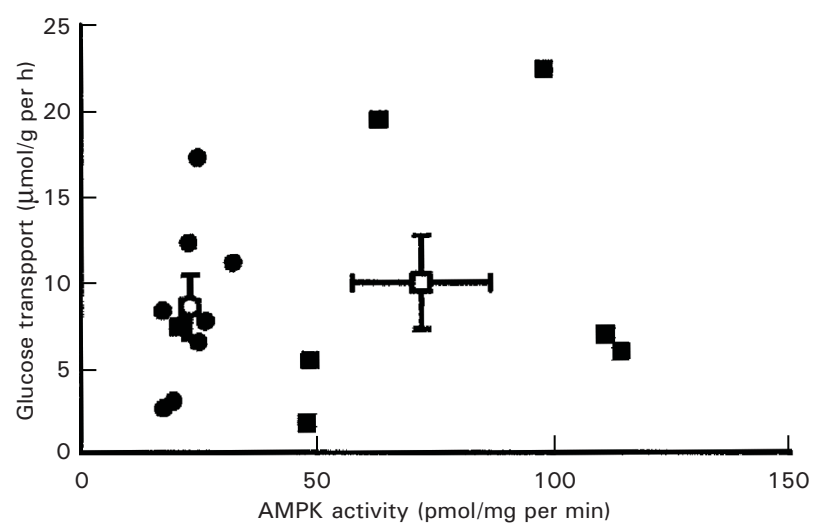

Fig. 2. The relationship between AMP-activated protein kinase (AMPK) activity and glucose transport during contraction in slowtwitch muscles (soleus). Individual data are shown for muscles with high precontraction glycogen levels $(\bullet)$ and muscles with low precontraction muscle glycogen levels $(\boldsymbol{\square}) .(\bigcirc, \square)$, Corresponding mean values with their standard errors represented by vertical and horizontal bars. (Reproduced, with permission, from Derave et al. 2000.) 
glucose transport. On the other hand, this study also shows that when muscles are electrically stimulated to contract, glucose transport is only inhibited by approximately $30 \%$ in the kinase-dead muscles compared with the wild-type muscle. This finding may suggest that contraction-induced glucose transport is partly dependent on AMPK. Thus, during contractions in vitro some extent of hypoxia, or at least perturbation of the energy status of the muscle, exists that might activate the feedback stimulation of glucose transport via activation of AMPK (Fig. 1). Obviously this pathway will not be activated in the kinase-dead muscles, even though the $\mathrm{Ca}^{2+}$-activated contraction pathway may be unaffected. Although this distinction between the two pathways may be a little tenuous, it probably has some merit in understanding the results obtained in studies involving exercise or contraction. The concept of two different pathways also helps to explain the apparent variation in the role of the different signalling molecules in different exercise conditions. Thus, from this model (Fig. 1) it would be expected that the role of the AMPKactivated feedback pathway would be greatest in severe exercise or contraction in which the energy status of the muscle is compromised, whereas the $\mathrm{Ca}^{2+}$-related feedforward pathway would dominate during less-intense exercise or contraction when AMPK is not activated or only moderately activated (Wojtaszewski et al. 2000).

The use of kinase-dead animals ( $\mathrm{Mu}$ et al. 2001) indicates only whether AMPK activity is important for a given biological response but cannot provide any information as to which of the AMPK isoforms might be involved. For this purpose, isoform-specific knock-out of AMPK can be used. AMPK is a heterotrimeric protein consisting of a catalytic $\alpha$ subunit and two regulatory subunits $(\beta$ and $\gamma)$. In skeletal muscle the $\alpha$ and $\beta$ subunits each exist in two isoforms $\left(\alpha_{1}\right.$ and $\alpha_{2}, \beta_{1}$ and $\left.\beta_{2}\right)$ while the $\gamma$ subunit exists in three isoforms $\left(\gamma_{1}, \gamma_{2}\right.$ and $\gamma_{3}$; Stapleton et al. 1996). The present authors, in collaboration with Vaulont and her colleagues in Paris, have recently carried out studies using $\alpha 1$ AMPK and $\alpha 2$ AMPK knock-out mice. The findings suggest that glucose transport is not affected during in vitro electrical stimulation of fast- or slow-twitch skeletal muscle from either $\alpha 1$ or $\alpha 2$ AMPK knock-out mice when compared with muscle from wild-type mice (Jørgensen et al. 2004). This finding is particularly interesting in relation to $\alpha 2$ AMPK knock-out mice in which the contraction-induced increase in AMPK-Thr ${ }^{172}$ phosphorylation has been shown to be markedly lower than that in the wild-type muscle. Thus, these data suggest either that expression of one AMPK isoform is sufficient to increase glucose transport during contraction, or that AMPK is not necessary for contraction-induced muscle glucose transport.

Another interesting finding derived from studies of AMPK knock-out mice is that AICAR-induced glucose transport and AMPK activation are completely abolished by $\alpha 2$ AMPK knock-out but unaffected by $\alpha 1$ AMPK knock-out (Jørgensen et al. 2004). Interestingly, in the muscle from the $\alpha 2$ AMPK knock-out mouse AICAR induces a doubling of $\alpha 1$ AMPK activity while glucose transport is completely unaffected. Thus, in muscle from $\alpha 2$ AMPK knock-out mice, in which a comparable increase in $\alpha 1$ AMPK activity occurs during contractions, it is unlikely that this increase in $\alpha 1$ AMPK activity has an effect on glucose transport during contraction. If this absence of an effect is confirmed, then the normal contraction-induced glucose transport in muscle from $\alpha 2$ AMPK knock-out mice (in which the AMPK activity stems from the $\alpha 1$ isoform) indicates that AMPK is not necessary for a normal increase in muscle glucose transport during contraction.

\section{Mitogen-activated protein kinases}

The mitogen-activated protein kinases (MAPK) belong to a superfamily consisting of at least three parallel and distinct MAPK pathways, which include the extracellular-regulated kinases and the two stress-activated protein kinase cascades, c-jun N-terminal kinase and p38 (for review, see Widegren et al. 2001). Recently, a potential role of p38 MAPK in contraction and insulin stimulation of glucose transport in skeletal muscle has been suggested (for review, see Furtado et al. 2003). Based on studies with p38 MAPK inhibitors and cell lines overexpressing a dominant negative p38 MAPK, it has been suggested that the p38 MAPK may be involved in increasing glucose transport by activating the GLUT4 transporter, although p38 MAPK does not seem to be involved in GLUT4 translocation to the cell surface. As p38 MAPK is activated by muscle contraction (Widegren et al. 2001) it might be involved in activating glucose transport during exercise.

\section{Conclusion}

Contraction-induced glucose uptake seems to be regulated by Ca-related mechanisms as well as mechanisms related to the metabolic status of the muscle. Elevated intracellular $\mathrm{Ca}^{2+}$ levels during contraction activate signalling pathways that may include CaMKII and PKC, whereas AMPK is activated when the metabolic status of the muscle is compromised. The p38 MAPK may also be involved in contraction-induced glucose transport. Evidence from mice in which a dominant negative AMPK is overexpressed suggests that AMPK may be responsible for $30-40 \%$ of contraction-induced muscle glucose transport, whereas recent results indicate that knock-out of either $\alpha 2$ or $\alpha 1$ AMPK does not affect contraction-induced muscle glucose transport. Thus, although there is little doubt that pharmacological activation of AMPK leads to increased glucose transport in resting skeletal muscle, direct evidence for the involvement of AMPK in glucose transport during muscle contraction is limited.

\section{Acknowledgements}

The present review was supported by grants from the Danish National Research Foundation (\#504-12), The Copenhagen Muscle Research Centre, The Media and Grants Secretariat of the Danish Ministry of Culture, the Danish Diabetes Association, the Novo Nordisk Foundation and a Research \& Technological Development Project (QLG1CT-2001-01488) funded by the European Commission. 
J.F.P.W. was supported by a Halla Møller fellowship from the Novo-Nordisk Research Foundation. J.N.N. was supported by a postdoctoral fellowship from the Carlsberg Foundation.

\section{References}

Beeson M, Sajan MP, Dizon M, Grebenev D, Gomez-Daspet J, Miura A, Kanoh Y, Powe J, Bandyopadhyay G, Standaert ML \& Farese RV (2003) Activation of protein kinase C-zeta by insulin and phosphatidylinositol-3,4,5-(PO4)3 is defective in muscle in type 2 diabetes and impaired glucose tolerance: Amelioration by rosiglitazone and exercise. Diabetes 52, 1926-1934.

Chen HC, Bandyopadhyay G, Sajan MP, Kanoh Y, Standaert M, Farese RV Jr \& Farese RV (2002) Activation of the ERK pathway and atypical protein kinase $\mathrm{C}$ isoforms in exercise- and aminoimidazole-4-carboxamide-1-beta-D-riboside (AICAR)stimulated glucose transport. Journal of Biological Chemistry 277, 23554-23562.

Chen ZP, McConell GK, Michell BJ, Snow RJ, Canny BJ \& Kemp BE (2000) AMPK signaling in contracting human skeletal muscle: acetyl-CoA carboxylase and NO synthase phosphorylation. American Journal of Physiology 279, E1202-E1206.

Cleland PJ, Appleby G, Rattigan S \& Clark M (1989) Exerciseinduced translocation of protein kinase $\mathrm{C}$ and production of diacylglycerol and phosphatidic acid in rat skeletal muscle in vivo. Journal of Biological Chemistry 264, 17704-17711.

Derave W, Ai H, Ihlemann J, Witters LA, Kristiansen S, Richter EA \& Ploug T (2000) Dissociation of AMP-activated protein kinase activation and glucose transport in contracting slowtwitch muscle. Diabetes 49, 1281-1287.

Derave W, Lund S, Holman GD, Wojtaszewski J, Pedersen O \& Richter EA (1999) Contraction-stimulated muscle glucose transport and GLUT-4 surface content are dependent on glycogen content. American Journal of Physiology 277, E1103-E1110.

Farese RV (2002) Function and dysfunction of aPKC isoforms for glucose transport in insulin-sensitive and insulin-resistant states. American Journal of Physiology 283, E1-E11.

Furtado LM, Poon V \& Klip A (2003) GLUT4 activation: thoughts on possible mechanisms. Acta Physiologica Scandinavica 178, 287-296.

Gollnick P, Pernow B, Essén B, Jansson E \& Saltin B (1981) Availability of glycogen and plasma FFA for substrate utilization in leg muscle of man during exercise. Clinical Physiology 1, 27-42.

Hayashi T, Hirshman MF, Kurth EJ, Winder WW \& Goodyear LJ (1998) Evidence for 5'AMP-activated protein kinase mediation of the effect of muscle contraction on glucose transport. Diabetes 47, 1369-1373.

Hespel P \& Richter EA (1990) Glucose uptake and transport in contracting, perfused rat muscle with different pre-contraction glycogen concentrations. Journal of Physiology (London) 427, 347-359.

Holloszy J \& Narahara H (1967) Enhanced permeability to sugar associated with muscle contraction. Journal of General Physiology 50, 551-562.

Holloszy JO \& Narahara HT (1965) Studies of tissue permeability. Journal of Biological Chemistry 240, 3493-3500.

Ihlemann J, Galbo H \& Ploug T (1999a) Calphostin C is an inhibitor of contraction, but not insulin-stimulated glucose transport, in skeletal muscle. Acta Physiologica Scandinavica 167, 69-75.
Ihlemann J, Ploug T \& Galbo H (2001) Effect of force development on contraction induced glucose transport in fast twitch rat muscle. Acta Physiologica Scandinavica 171, 439-444.

Ihlemann J, Ploug T, Hellsten Y \& Galbo H (1999b) Effect of tension on contraction-induced glucose transport in rat skeletal muscle. American Journal of Physiology 277, E208-E214.

Ihlemann J, Ploug T, Hellsten Y \& Galbo H (2000) Effect of stimulation frequency on contraction-induced glucose transport in rat skeletal muscle. American Journal of Physiology 279, E862-E867.

Jørgensen SB, Viollet B, Andreelli F, Frøsig C, Birk JB, Schjerling P, Vaulont S, Richter EA \& Wojtaszewski JFP (2004) Knockout of the $\alpha_{2}$ but not $\alpha_{1} 5^{\prime}$-AMP-activated protein kinase isoform abolishes 5-aminoimidazole-4-carboxamide1- $\beta$-4-ribofuranoside but not contraction-induced glucose uptake in skeletal muscle. Journal of Biological Chemistry 279, 1070-1079.

Merrill GF, Kurth EJ, Hardie DG \& Winder WW (1997) AICA riboside increases AMP-activated protein kinase, fatty acid oxidation, and glucose uptake in rat muscle. American Journal of Physiology 273, E1107-E1112.

Mu J, Brozinick JT Jr, Valladares O, Bucan M \& Birnbaum MJ (2001) A role for AMP-activated protein kinase in contractionand hypoxia-regulated glucose transport in skeletal muscle. Molecular Cell 7, 1085-1094.

Nielsen JN, Frøsig C, Sajan M, Miura A, Standaert ML, Graham DA, Wojtaszewski JFP, Farese RV \& Richter EA (2003) Increased atypical protein kinase $\mathrm{C}$ activity in endurancetrained human skeletal muscle. Biochemical and Biophysical Research Communications 312, 1147-1153.

Nielsen JN, Wojtaszewski JF, Haller RG, Hardie DG, Kemp BE, Richter EA \& Vissing J (2002) Role of 5'AMP-activated protein kinase in glycogen synthase activity and glucose utilization: insights from patients with McArdle's disease. Journal of Physiology (London) 541, 979-989.

Perrini S, Henriksson J, Zierath JR \& Widegren U (2004) Exercise-induced protein kinase $\mathrm{C}$ isoform-specific activation in human skeletal muscle. Diabetes 53, 21-24.

Ploug T, van Deurs B, Ai H, Cushman SW \& Ralston E (1998) Analysis of GLUT4 distribution in whole skeletal muscle fibers: identification of distinct storage compartments that are recruited by insulin and muscle contractions. Journal of Cell Biology 142, 1429-1446.

Richter EA, Cleland PJ, Rattigan S \& Clark MG (1987) Contraction-associated translocation of protein kinase $\mathrm{C}$ in rat skeletal muscle. FEBS Letters 217, 232-236.

Richter EA \& Galbo H (1986) High glycogen levels enhance glycogen breakdown in isolated contracting skeletal muscle. Journal of Applied Physiology 61, 827-831.

Rose AJ \& Hargreaves M (2003) Exercise increases $\mathrm{Ca}^{++}$ calmodulin-dependent protein kinase II activity in human skeletal muscle. Journal of Physiology (London) 553, 303-309.

Stapleton D, Mitchelhill KI, Gao G, Widmer J, Michell BJ, Teh T, House CM, Fernandez CS, Cox T, Witters LA \& Kemp BE (1996) Mammalian AMP-activated protein kinase subfamily. Journal of Biological Chemistry 271, 611-614.

Vavvas D, Apazidis A, Saha AK, Gamble J, Patel A, Kemp BE, Witters LA \& Ruderman NB (1997) Contraction-induced changes in acetyl-CoA carboxylase and 5'-AMP-activated kinase in skeletal muscle. Journal of Biological Chemistry 272, 13255-13261.

Widegren U, Ryder JW \& Zierath JR (2001) Mitogen-activated protein kinase signal transduction in skeletal muscle: effects of exercise and muscle contraction. Acta Physiologica Scandinavica 172, 227-238.

Winder WW \& Hardie DG (1996) Inactivation of acetyl-CoA carboxylase and activation of AMP-activated protein kinase in 
muscle during exercise. American Journal of Physiology 270, E299-E304.

Winder WW \& Hardie DG (1999) AMP-activated protein kinase, a metabolic master switch: possible roles in type 2 diabetes. American Journal of Physiology 277, E1-E10.

Wojtaszewski JF, Jorgensen SB, Hellsten Y, Hardie DG \& Richter EA (2002) Glycogen-dependent effects of 5-aminoimidazole-4-carboxamide (AICA)-riboside on AMPactivated protein kinase and glycogen synthase activities in rat skeletal muscle. Diabetes 51, 284-292.

Wojtaszewski JF, MacDonald C, Nielsen JN, Hellsten Y, Hardie DG, Kemp BE, Kiens B \& Richter EA (2003) Regulation of 5'AMP-activated protein kinase activity and substrate utilization in exercising human skeletal muscle. American Journal of Physiology 284, E813-E822.

Wojtaszewski JF, Nielsen P, Hansen BF, Richter EA \& Kiens B (2000) Isoform-specific and exercise intensitydependent activation of 5'-AMP-activated protein kinase in human skeletal muscle. Journal of Physiology (London) 528, 221-226.
Wojtaszewski JFP, Laustsen JL \& Richter EA (1998) Contraction- and hypoxia-stimulated glucose transport in skeletal muscle is affected differently by wortmannin. Evidence for different signalling mechanisms. Biochimica et Biophysica Acta 1340, 396-404.

Wright CD, Hucker KA, Holloszy JO \& Han DO (2004) $\mathrm{Ca}^{++}$ and AMPK both mediate stimulation of glucose transport by muscle contractions. Diabetes 53, 330-335.

Yokokura H, Terada O, Naito Y, Sugita R \& Hidaka H (1997) Cascade activation of the calmodulin kinase family. Advances in Second Messenger and Phosphoprotein Research 31, 151-157.

Youn JH, Gulve EA, Henriksen EJ \& Holloszy JO (1994) Interaction between effects of W-7, insulin and hypoxia on glucose transport in skeletal muscle. American Journal of Physiology 267, R888-R894.

Youn JH, Gulve EA \& Holloszy JO (1991) Calcium stimulates glucose transport in skeletal muscle by a pathway independent of contraction. American Journal of Physiology 260, C555-C561. 